\title{
A Rare Case of Invasive Apocrine Carcinoma of the Breast with Unusual Radiologic Findings
}

\author{
Ji Min Kim, ${ }^{1}$ Shin Young Kim, ${ }^{1, *}$ Mee Hye Oh, ${ }^{2}$ and Jong Eun Lee ${ }^{3}$ \\ ${ }^{1}$ Department of Radiology, Cheonan Hospital, Soonchunhyang University College of Medicine, Cheonan, Korea \\ ${ }^{2}$ Department of Pathology, Cheonan Hospital, Soonchunhyang University College of Medicine, Cheonan, Korea \\ ${ }^{3}$ Department of Surgery, Cheonan Hospital, Soonchunhyang University College of Medicine, Cheonan, Korea \\ "Corresponding author: Shin Young Kim, Department of Radiology, Soonchunhyang University College of Medicine, Cheonan Hospital, 31 Soonchunhyang 6-gil, Dongnam-gu, \\ Chungcheongnam-do Cheonan, Korea. Tel: +82-415703515, Fax: +82-415703516, E-mail: c87093@schmc.ac.kr
}

Received 2015 December 14; Revised 2016 February 20; Accepted 2016 May 02.

\begin{abstract}
Invasive apocrine carcinoma (IAC) of the breast is a rare subtype of breast malignancy. Its incidence is not well known, but it is approximately less than $1 \%$ to $4 \%$. For these reasons, there are few reports and little information on the radiologic appearance of IAC. Furthermore, most of the case reports show malignant features which are similar to invasive ductal carcinoma (IDC). We present a rare case of IAC without typical malignant feature on mammography, and ultrasonography (USG). Imaging findings on computed tomography (CT), magnetic resonance imaging (MRI), and 18F-fluorodeoxyglucose (FDG) positron emission tomography (PET)/CT are also presented. The nodule in our case showed a relatively benign feature on USG and it is the first case of IAC with unusual findings. Therefore, this report may encourage radiologists to consider the malignant potential and perform pathologic correlation even if a newly developed nodule does not present with a typical malignant feature on USG.
\end{abstract}

Keywords: Breast, Breast Neoplasms, Apocrine Glands, Mammography, Ultrasonography

\section{Introduction}

Apocrine gland lesions of the breast have various benign and malignant features. Malignant apocrine lesions of the breast include apocrine ductal carcinoma in situ (ADCIS) and invasive apocrine carcinoma (IAC) (1). IAC is a distinctive and rare subtype of breast malignancy that consists of more than $90 \%$ of apocrine differentiation (1, 2 ). The incidence of IAC is reported as less than $1 \%$ to $4 \%$ of female invasive carcinoma (3). Microscopically, IAC shows identical architectural growth pattern as invasive ductal carcinoma (IDC) of no special type (NOS), differing only in cytologic appearance (4). In addition, IAC has a similar clinical presentation and overall patient survival rate similar to IDC of NOS (3). There are few case reports on radiologic findings of IAC, but all cases show comparable malignant appearance to IDC (5-8). Herein, we present a rare case of IAC with its multiple radiologic modality findings that suggests a benign rather than malignant feature on initial ultrasonography (USG) and mammography and the pathologic correlation.

\section{Case Presentation}

A 61-year-old woman presented to our hospital due to an abnormal finding on screening mammography. There was no palpable lesion, pain or nipple discharge on physical examination at the time of the visit. The patient had a history of excision and biopsy on her left breast 9 years before that confirmed fibroadenoma in our medical center.

Mammography showed a newly developed partly indistinct oval isodense nodule in the upper center of the right breast without any microcalcification (Figure 1). Therefore, we categorized this lesion as breast imaging reporting and data system (BI-RADS) category 0 and recommended breast USG. USG images showed an oval circumscribed hypoechoic nodule about $1.0 \mathrm{~cm}$ in size with an internal cystic portion and peripheral vascularity in the upper center portion of the right breast that correlated with the mammographic finding (Figure 2). We assumed this lesion as BI-RADS category 4A. Invasive carcinoma was diagnosed based on findings from USG guided core needle biopsy. The pathologist suggested apocrine, oncocytic and glycogen-rich carcinomas for differential diagnosis.

Chest computed tomography (CT) showed a mild enhanced lesion in the upper center and outer portion of the right breast, but there was no evidence of lung metastasis (Figure 3). Breast dynamic magnetic resonance imaging (MRI) showed an approximately $1.1 \mathrm{~cm}$ sized oval irregular heterogeneous enhanced nodule in the right upper center portion. This nodule showed an early rim enhancement and delayed wash-out pattern (Figure 4). We catego- 


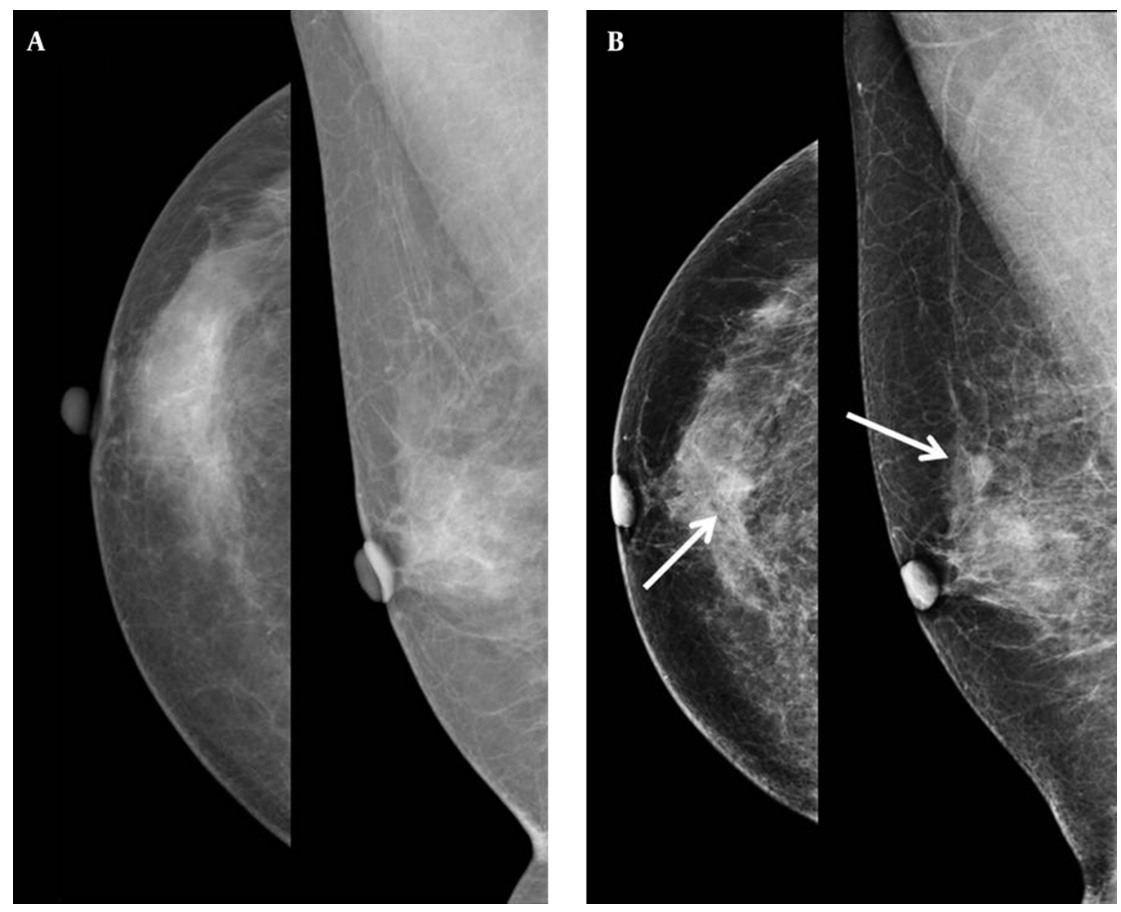

Figure 1. A 61-year-old woman with abnormal findings on screening mammography. A, There was no remarkable finding in mammography in December 2011. B, Follow-up mammography in March 2015 shows a newly developed partly indistinct oval isodense nodule in the upper center of the right breast (arrows).
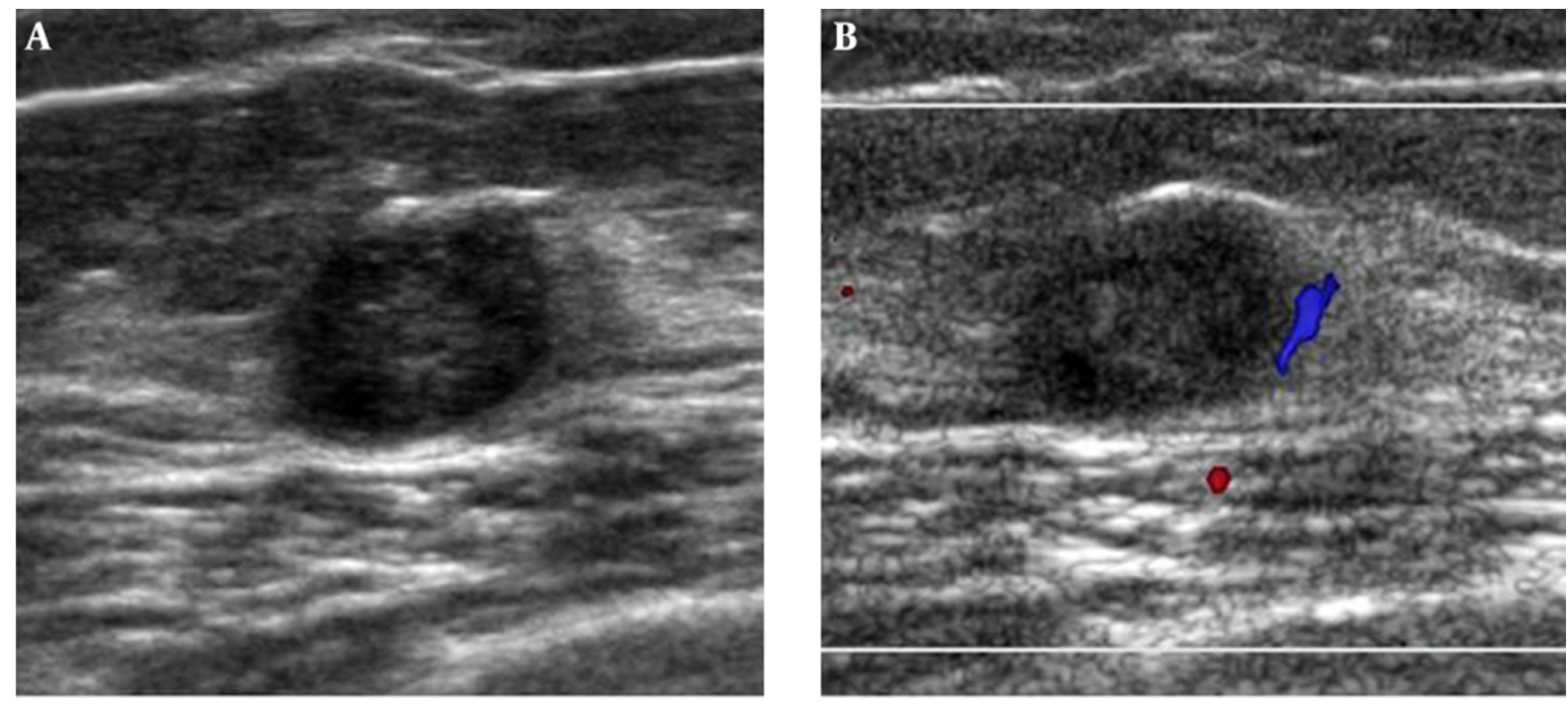

Figure 2. A, Ultrasonography reveals a $1.0 \mathrm{~cm}$ sized oval circumscribed hypoechoic nodule with an internal cystic portion in the upper center portion of the right breast. B, Peripheral vascularity is present in the nodule.

rized this lesion as BI-RADS 6. Additional positron emission tomography (PET)/CT was performed and the nodule showed increased 18F-fluorodeoxyglucose (FDG) uptake of about 1.63 in maximum standardized uptake value (max
SUV) (Figure 5). There was no evidence of distant metastasis on PET/CT.

Finally, invasive apocrine carcinoma was confirmed on lumpectomy of the right breast. Pathologic study re- 
vealed abundant eosinophilic cytoplasm with prominent nuclei in the tumor (Figure 6A). Immunohistochemical stain showed estrogen receptor (ER), progesterone receptor (PR), human epidermal growth factor receptor 2 negativities, and gross cystic disease protein fluid-15 (GCDPF15) positivity (Figure 6B). Cyclophosphamide, methotrexate and fluorouracil combination chemotherapy was administered for the patient.

\subsection{Ethical Statement}

This study was approved by the institutional review board of the Soon Chun Hyang University Cheonan Hospital. It has been performed in accordance with the ethical standards laid down in the 1964 Declaration of Helsinki and its later amendments. The institutional review board of this university waived the need to obtain informed consent.

\section{Discussion}

The change of breast epithelium to apocrine epithelium is relatively common resulting in a wide range of benign and malignant pathologies (1). Malignant lesions due to apocrine change of the breast can be divided into ADCIS and IAC (1). IAC was initially described by Krompecher in

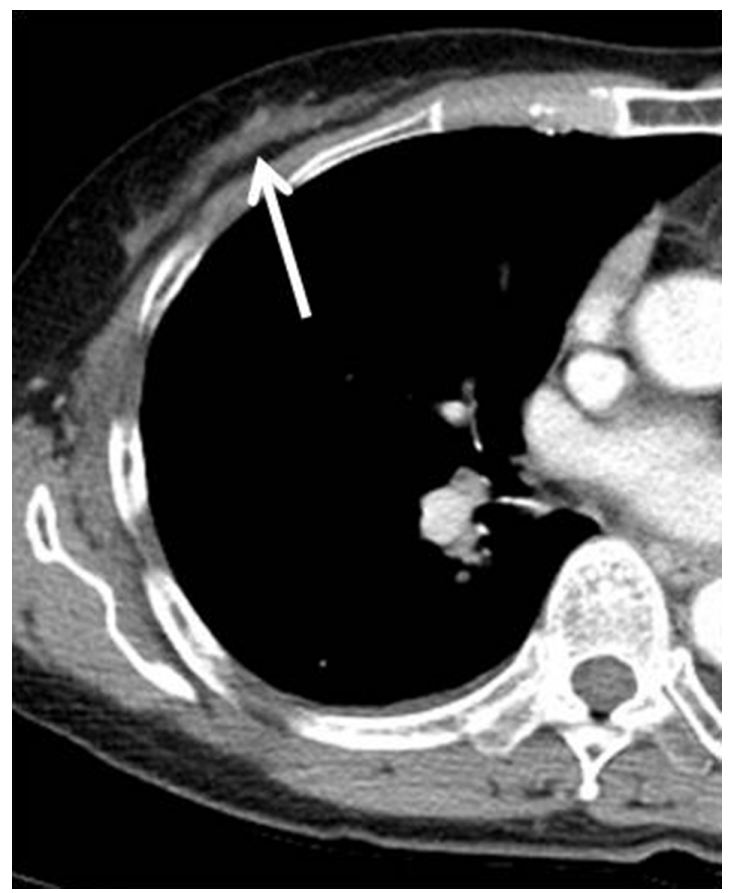

Figure 3. Computerized tomography of the chest shows mild enhancement of the nodule (arrow).
1916, and is now defined as more than $90 \%$ of apocrine differentiated neoplastic cells $(1,9)$. The incidence of IAC is reportedly variable from less than $1 \%$ to $4 \%$ (8). However, the definite incidence of IAC remains unknown currently, because there is no standardized consensus on the standard diagnostic criteria for IAC, despite the diagnostic criteria of Japaze et al. in 2005 (3). IAC is thought to have similar clinical, macroscopic and histological features as IDC of NOS (1, $3,4)$. Both diseases have a greater likelihood in women over 40 (10). In general, hormonal factors such as nulliparity, first child after 30 , early menarche, and late menopause increase the risk of breast cancer $(1,10)$. Continuous stimulation of sex hormones such as androgen seems to be related to the development of apocrine metaplasia in the breast (1). Physical examination varies from asymptomatic to the presence of a palpable mass with or without bloody nipple discharge $(6,10)$. The death rate of recurrent breast cancer occurs in 5 to $8 \%$ of the cases, which is similar to IDC (10).

Microscopically, IAC presents almost the same architectural growth pattern as IDC of NOS, but it shows difference only in its cytological appearance (8). The cells are characterized by typical apocrine features such as abundant, eosinophilic granular cytoplasms, and prominent or multiple nucleoli (3). According to emerging evidence, apocrine carcinomas tend to show ER, PR and Bcl-2 negativity and androgen receptor positivity and expression of GCDPF15 (1). A histopathological report indicated that GCDPF-15 positivity was correlated with small size, negative lymph node, and lower histologic grade of the tumor. Likewise, our case showed the same immunohistochemical stain result (1). Several reports suggest that apocrine carcinomas show a unique response to androgen (such as fluoxymesterone) administration as a part of treatment, but there is no standardized treatment option for IAC (8).

Radiologic appearance of IAC is difficult to classify by the available modalities because of the limited information. Gilles et al. reported that most of the mammographic findings of IAC are associated with microcalcification with no different features from IDC (6). Also, Onoue et al. reported a case of IAC that showed an oval circumscribed hyperdense nodule with microcalcification in mammography (7). Gokalp et al. showed an irregular shaped IAC with microcalcification (5). Seo et al. presented five case reports with mammographic findings in three cases (8). One case showed an irregular, partly indistinct hyperdense nodule and the other presented as an asymmetry (8). In contrast, our mammography showed a partly indistinct oval isodense nodule without any microcalcification. According to several previous case reports, IAC shows variable sonographic appearances including irregular shaped, non-circumscribed solid mass with heterogeneous internal echo pattern $(5,7,8)$. Onoue et al. reported two oval hy- 

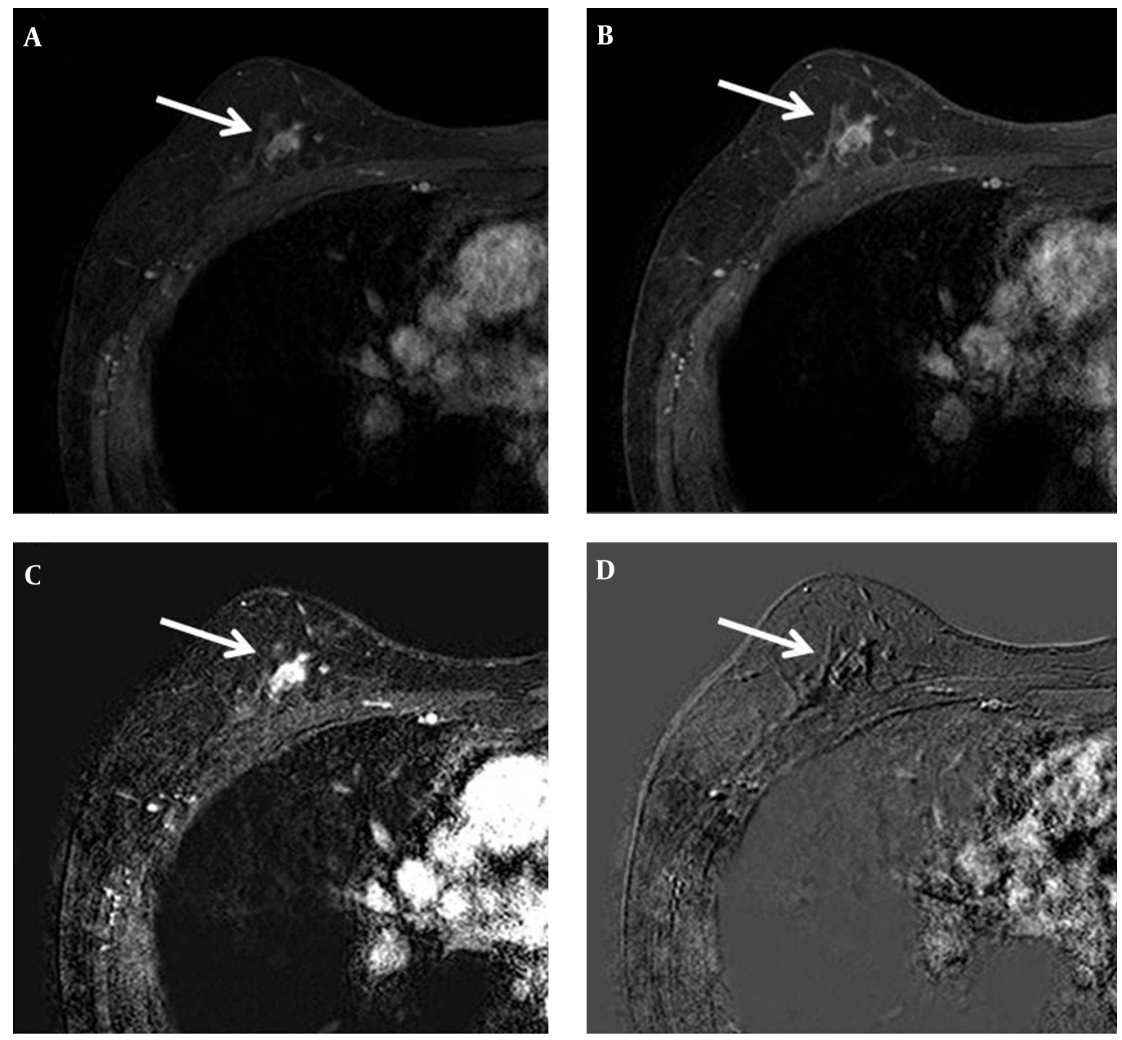

Figure 4. MRI was performed using the 3.0-T system. An oval irregular nodule approximately $1.1 \mathrm{~cm}$ in size is detected in the upper center portion of the right breast. A, Axial early ( 2 minutes) contrast-enhanced and B, Delayed ( 6 minutes) contrast-enhanced axial T1-weighted images after bolus administration of gadolinium-based contrast agent show rim enhancement. C, Axial subtraction image reveals rapid initial enhancement and D, Reversed subtraction image reveals delayed wash out (arrow).

poechoic cysts with hypoechoic papillary projection with stromal invasion in some areas. (7). Gokalp et al. reported

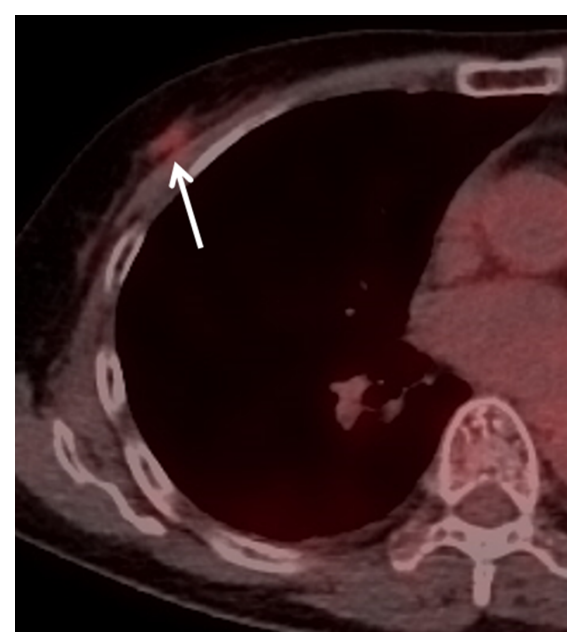

Figure 5. PET/CT shows increased FDG uptake (max SUV 1.63) in the nodule (arrow). cases that showed two different IACs; one with a solid spiculated angular shape and the other as a complex cyst containing solid components and thick septa (5). Seo et al. reported five cases that all showed irregular solid masses with heterogeneous internal echo patterns and noncircumscribed margins (8). In this case, USG showed an oval circumscribed hypoechoic nodule with an internal cystic change. To the best of our knowledge, there is only one case report on MRI findings of IAC. Seo et al. reported IAC with rapid, heterogeneous enhancement during the initial phase and washout in the delayed phase (8). Our MRI showed oval spiculated nodule with rapid, rim enhancement and delayed washout pattern that was similar to the case reported by Seo et al. (8). Most authors agree that it is difficult to differentiate IAC from IDC by radiologic appearance alone (5-8). Moreover, there is no established specific radiologic finding of IAC yet.

As described above, several previous case reports on radiologic findings of IAC demonstrated relative malignant features; furthermore, it is accepted that it is not different from IDC in radiologic appearance (5-8). However, our 


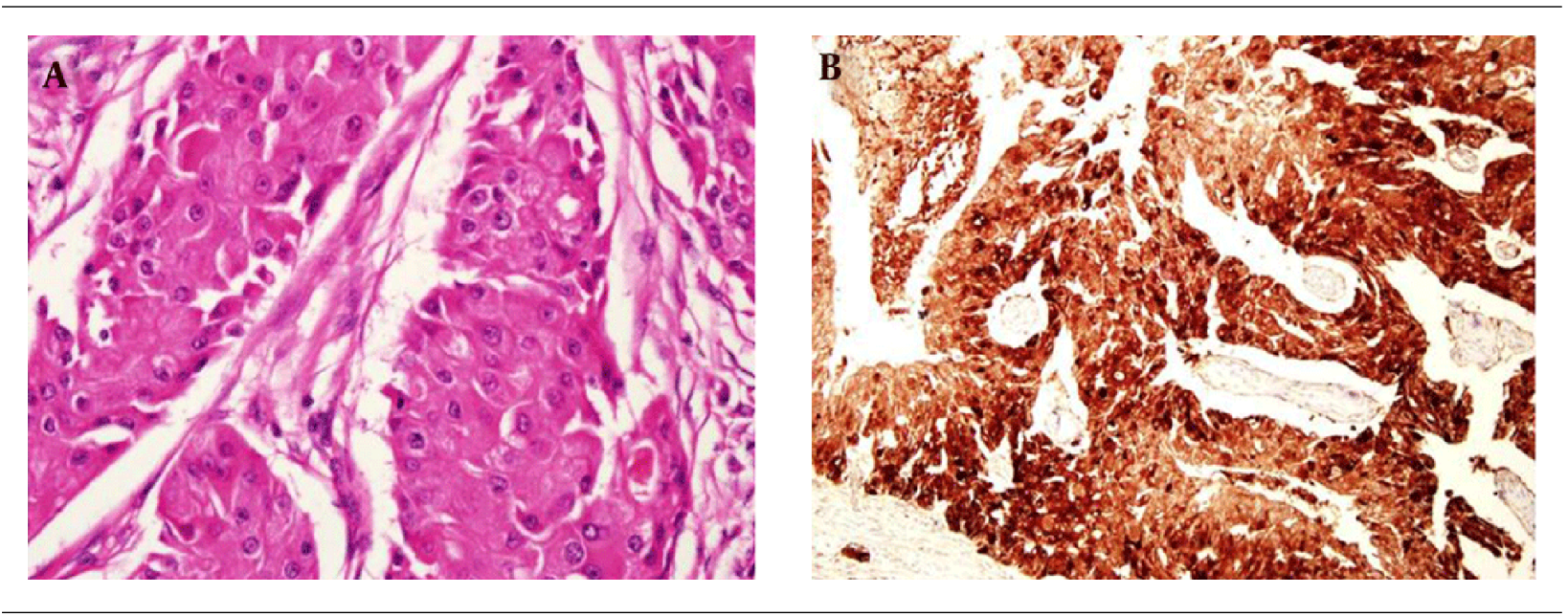

Figure 6. Microscopic examination shows A, Abundant eosinophilic cytoplasm with prominent nucleoli (H\&E, $\times 400)$ and B, Diffuse strong positive reaction for GCDFP-15 in the tumor $(\times 200)$.

case of IAC showed comparatively unusual appearance in mammography and USG. In addition, it did not contain microcalcification in mammography, as in previous cases. To the best of our knowledge, this is the first case report on IAC presenting as non typical malignant feature. In conclusion, radiologists should consider the malignant potential of newly developed nodule and evaluate the lesion pathologically despite the benign feature seen with radiologic imaging modalities.

\section{Footnotes}

Authors' Contributions: Ji Min Kim drafted the manuscript. Ji Min Kim and Shin Young Kim performed critical revision of the manuscript for important intellectual content. Mee Hye Oh, Shin Young Kim and Jong Eun Lee were responsible for administrative, technical, and material support. Shin Young Kim supervised the study.

Conflict of Interest: Ji Min Kim, Shin Young Kim, Mee Hye Oh and Jong Eun Lee declare that they have no conflict of interest.

Financial Disclosure: The manuscript has not been published before or is not under consideration for publication anywhere else and has been approved by all co-authors in the title page.

Funding/Support: This work was supported by the Soonchunhyang University research fund.

\section{References}

1. Gerhard R, Costa JL, Schmitt F. Benign and malignant apocrine lesions of the breast. Expert Rev Anticancer Ther. 2012;12(2):215-21. doi: 10.1586/era.11.213. [PubMed: 22316369].

2. Vranic S, Schmitt F, Sapino A, Costa JL, Reddy S, Castro M, et al. Apocrine carcinoma of the breast: a comprehensive review. Histol Histopathol. 2013;28(11):1393-409. [PubMed: 23771415].

3. Japaze H, Emina J, Diaz C, Schwam RJ, Gercovich N, Demonty $\mathrm{G}$, et al. 'Pure' invasive apocrine carcinoma of the breast: a new clinicopathological entity?. Breast. 2005;14(1):3-10. doi: 10.1016/j.breast.2004.06.003. [PubMed: 15695074].

4. Eusebi V, Millis RR, Cattani MG, Bussolati G, Azzopardi JG. Apocrine carcinoma of the breast. A morphologic and immunocytochemical study. Am J Pathol. 1986;123(3):532-41. [PubMed: 3717305].

5. Gokalp G, Topal U, Haholu A, Kizilkaya E. Apocrine carcinoma of the breast: mammography and ultrasound findings. European Journal of Radiology Extra. 2006;60(2):55-9.

6. Gilles R, Lesnik A, Guinebretiere JM, Tardivon A, Masselot J, Contesso $G$, et al. Apocrine carcinoma: clinical and mammographic features. Radiology. 1994;190(2):495-7. doi: 10.1148/radiology.190.2.8284405. [PubMed: 8284405]

7. Onoue S, Katoh T, Chigira H, Matsuo K, Suzuki M, Shibata Y, et al. A case of apocrine carcinoma of the breast presenting as two cysts. Breast cancer. 1997;4(3):193-6.

8. Seo KJ, An YY, Whang IY, Chang ED, Kang BJ, Kim SH, et al. Sonography of Invasive Apocrine Carcinoma of the Breast in Five Cases. Korean J Radiol. 2015;16(5):1006-11. doi: 10.3348/kjr.2015.16.5.1006. [PubMed: 26357495].

9. Krompecher E. Zur Histogenese und Morphologie der Cystenmamma (maladie kystique reclus, cystadenoma Schimmelbuscin, mastitis chronica cystica Konig) des intrakanalikularen Kystadenomas und der Kystadenokarzinome der Brustdruse (Hidrokystoma, kystadenoma, Hidrokystadenocarzinoma mammae) [in French]. Beitr Pathol Anat. 1916;62(1):403-10.

10. Bedford T, Alperstein A, Nathani Y, Marx R, DeVito P. A rare presentation of triple-negative apocrine breast carcinoma with metastases. J Surg Case Rep. 2014;2014(5) doi: 10.1093/jscr/rju045. [PubMed: 24876514]. 